\title{
Outcomes of Hospitalized COVID-19 Patients Receiving Renin Angiotensin System Blockers and Calcium Channel Blockers
}

\author{
Tatvam T. Choksi ${ }^{a}$ Hui Zhang ${ }^{b}$ Thomas Chen $^{a}$ Nikhil Malhotra $^{a}$ \\ aDepartment of Hospital Medicine, The University of Chicago Medical Center, Chicago, IL, USA; ${ }^{\mathrm{b}}$ The Center for \\ Health and the Social Sciences, The University of Chicago, Chicago, IL, USA
}

\section{Keywords}

Angiotensin-converting enzyme inhibitors · Angiotensin receptor blockers $\cdot$ Calcium channel blockers $\cdot$ Intensive care unit admission · Mortality

\begin{abstract}
Introduction: Use of certain antihypertensive medications has been an area of interest during the COVID-19 pandemic, and several hypotheses have been developed regarding the effects of renin-angiotensin system blockers as well as calcium channel blockers in those infected with COVID-19. We seek to determine the association between exposure to ACEI, ARB, and CCB and outcomes in those admitted to the hospital with COVID-19 infection. Methods: This retrospective cohort study included 841 adult patients hospitalized with COVID-19 infection at the University of Chicago Medical Center between March 25 and June 22, 2020. Out of these 841,453 patients had a personal history of hypertension. For the first part, we evaluated primary outcomes of in-hospital mortality and ICU admission in hospitalized COVID-19 patients based on their exposure to particular medications regardless of a personal history of hypertension and compared them with those who were not on these medications. For the second part, we evaluated the aforementioned outcomes in
\end{abstract}

453 patients with a personal history of hypertension based on their medication exposure. Secondary outcomes of length of stay, readmission rate, and new-onset dialysis requirement were also compared across the study groups. $R \boldsymbol{e}$ sults: Out of 841 patients, 111 (13.19\%) were on ACEI/ARB (median age: 66.1, SD 15.4;52.25\% females) and $730(86.80 \%)$ were not on them (median age: 56.6, SD 20.3; $50.14 \%$ females), while 277 (32.93\%) used CCB (median age: 64.6, SD $15.2 ; 57.04 \%$ females) and 564 (67.06\%) did not use CCB (median age: 54.6, SD 21.2; $47.16 \%$ females). After adjusting for demographics and covariates, neither ACEI/ARB nor CCB exposure was associated with any effect on mortality, but ACEI/ ARB exposure was associated with $42 \%$ reduction in risk of ICU admissions (OR 0.58, 95\% Cl $[0.35,0.95], p$ value 0.03 ). In addition, combined use of ACEI/ARB and CCB was associated with statistically significant (45\%) reduction in ICU admission (OR $0.55,95 \% \mathrm{Cl}[0.32,0.94], p$ value 0.029 ). Out of 453 patients with a personal history of hypertension, 85 (18.76\%) were taking ACEI/ARB (median age 65, SD 15.6; $56.47 \%$ females) and 368 (81.24\%) were not on ACEI/ARB (median age 62.8 , SD $16.4 ; 54.89 \%$ females), while 208 (45.92\%) out of 453 were on CCB (median age $65 ;$ SD $14.8 ; 60.1 \%$ females) and 245 (54.08\%) were not on CCB (median age 61.7, SD 17.3; $51.02 \%$ females). In the fully adjusted model in this group, ACEI use was associated with $71 \%$ reduction in in-house 
mortality (OR $0.29,95 \% \mathrm{Cl}[0.09,0.93], p$ value 0.03$)$. Discussion/Conclusion: Among all hospitalized patients with COVID-19 infection, exposure to ACEI/ARB, as well as combined exposure to ACEI/ARB and CCB, were associated with reduced incidence of ICU admissions. In those admitted patients who had a personal history of hypertension, there was a trend towards reduced in-hospital mortality in those exposed to ACEl.

(c) 2021 S. Karger AG, Basel

\section{Introduction}

Since the outbreak of COVID-19 infections in Hubei Province of China in December 2019, the novel coronavirus (SARS-CoV-2) has spread rapidly across the globe. It has affected $>90$ million people around the world and has been responsible for $>2$ million deaths till date [1]. As the disease is still relatively novel, data regarding the impact of underlying comorbidities and roles of chronic medical problems on the severity of COVID-19 infection are still emerging.

Age $>65$ years has been deemed as one of the important risk factors for severe COVID-19 infection [2]. Apart from age, certain underlying chronic medical conditions including cardiovascular diseases, chronic lung diseases, malignancies, and immunocompromised states appear to play an important role not only in increasing susceptibility to COVID-19 infections but also in affecting prognosis of these patients [3]. Hypertension is one of the most common comorbidities noted in adult population affected with COVID-19, and reports of patients with hypertension who tend to suffer poorer outcomes compared to those without hypertension have circulated since the beginning of the pandemic [4]. In line with aforementioned findings and based on certain experimental animal models, various hypotheses have been generated, and studies have been conducted evaluating safety of chronic exposure to certain cardiovascular medications in relation to COVID-19 infections.

This has been especially true for angiotensin-converting enzyme inhibitors (ACEI) and angiotensin receptor blockers (ARB) and lately has also involved calcium channel blockers (CCB) in those affected with COVID-19. ACEI, ARB, and CCB are some of the most commonly used antihypertensive medications. Apart from hypertension, ACEI and ARB are also associated with reduced morbidity and mortality in treatment of congestive heart failure, coronary artery disease, and CKD.

ACE- 2 receptor has been identified as one of the major receptors of entry for SARS-CoV-2 [5], and based on ex- perimental animal models suggesting ACEI/ARB upregulates ACE-2 in cell membranes [6-8], hypotheses had been generated that these medications may promote increased SARS-CoV-2 entry into cells and an increased risk for severe COVID-19 infection [9]. However, previous studies assessing the effects of ACEI/ARB on ACE-2 expression were not particularly done in lung tissue which is the presumed source of SARS-CoV-2 infection. A recent study by Wysocki et al. [10] showed no effect of ACEI and ARB administration in lung tissue ACE-2 expression. But, due to the importance of ACEI and ARB in routine clinical practice, articles have been published elaborating on the hypothesis of severe COVID-19 infection in those exposed to these medications [11-15], and several studies have been conducted to assess the true association. None of these have shown an increased risk of mortality or ICU admission in relation to ACEI/ARB [16-28]. Moreover, some favorable outcomes have been reported in a subset of these studies [20,26-28]. But, the majority of these studies have been conducted either in the predominant Caucasian population or in the Chinese population. Only 1 small study reported had predominant African American population, and it showed no association of outcomes related to ACEI or ARB use [19].

In the interim, interest has also started developing in terms of CCB use in patients infected with COVID-19. It has been established that the calcium ion is essential for viral entry, viral gene replication, and virion maturation and release in certain viruses [29, 30]. Navarese et al. [30] suggested that calcium plays an active role in inducing viral membrane fusion by altering the fusion peptide's structure and promotes infection in MERS-CoV, SARS$\mathrm{CoV}$, and SARS-CoV-2. In lieu of these, a few observational studies have been conducted which have reported potentially improved mortality and decrease in severity of COVID-19 infection with respect to CCB use [28, 3133]. But, the studies were either highly underpowered or included predominantly Asian or Caucasian population.

Essential hypertension is much more common in the African American population compared to other ethnic groups, and COVID-19 has affected the aforementioned group much more severely compared to others [34, 35]. Therefore, here, we aimed to evaluate the outcomes in hospitalized COVID-19 patients at the University of Chicago Medical Center in relation to the baseline use of $\mathrm{ACEI}, \mathrm{ARB}$, and $\mathrm{CCB}$ where the patient subgroup is predominantly composed of the African American patients. Moreover, we analyzed outcomes based on the combined exposure to ACEI/ARB and CCB use, which from our review of the literature has not been studied so far. 
Table 1. Baseline characteristics of hospitalized COVID-19 patients with respect to medications exposure

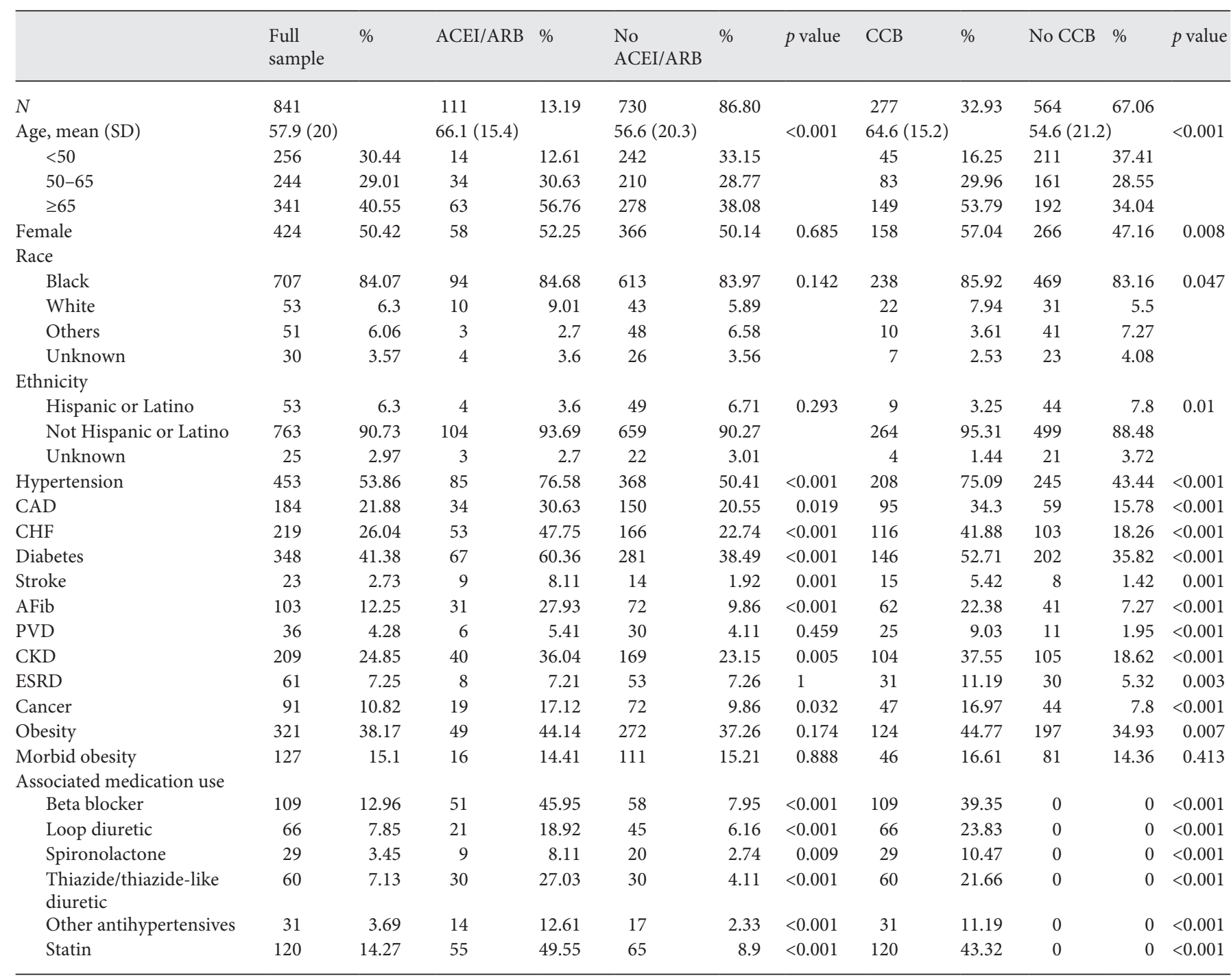

ACEI, angiotensin-converting enzyme inhibitors; ARB, angiotensin receptor blockers; CCB, calcium channel blockers.

\section{Methods}

Study Design and Participants

This retrospective cohort study included 841 patients with COVID-19 infection admitted to the University of Chicago Medical Center, Chicago, IL, USA. The study protocol was approved by the Institutional Review Board (IRB) Committee, and patient informed consent was waived by the IRB. COVID-19 diagnosis was determined by testing positive for the CDC 2019 novel coronavirus (2019-nCoV) real-time reverse transcriptionpolymerase chain reaction (RT-PCR) diagnostic panel detecting SARS-CoV-2 virus on nasopharyngeal swab/upper respiratory tract.

All adult patients aged 18 years and above who were hospitalized at the University of Chicago Medical Center with COVID-19 infection (as determined by positive SARS-CoV-2 testing) between March 15 and June 22, 2020, were included. Those whose medication history was not available were excluded.

\section{Data Collection}

Once IRB approval was obtained, electronic health records data within 1 year prior to COVID-19 testing were collected on all necessary variables. Data were collected on patient demographic information (age, gender, and race/ethnicity), diagnosis, and comorbidities (hypertension, diabetes mellitus, coronary artery disease, congestive heart failure, CKD stage 1-4, CKD stage 5/end-stage renal disease, cerebrovascular diseases [stroke/transient ischemic attacks], congestive heart failure, obesity, morbid obesity, atrial fibrillation, peripheral vascular diseases, and cancer). In addition, information on appropriate laboratory data, clinical data (vital 
Table 2. Baseline characteristics of hospitalized COVID-19 patients with a personal history of hypertension with regard to medication exposure

\begin{tabular}{|c|c|c|c|c|c|c|c|c|c|c|c|c|}
\hline & $\begin{array}{l}\text { Full } \\
\text { sample }\end{array}$ & $\%$ & ACEI/ARB & $\%$ & $\begin{array}{l}\text { No } \\
\text { ACEI/ARB }\end{array}$ & $\%$ & $p$ value & ССВ & $\%$ & No CCB & $\%$ & $p$ value \\
\hline$N$ & 453 & & 85 & & 368 & & & 208 & & 245 & & \\
\hline Age & $63.2(16.3)$ & & $65.0(15.6)$ & & $62.8(16.4)$ & & 0.326 & $65.0(14.8)$ & & $61.7(17.3)$ & & 0.049 \\
\hline$<50$ & 85 & 18.76 & 11 & 12.94 & 74 & 20.11 & & 31 & 14.9 & 54 & 22.04 & \\
\hline $50-65$ & 14 & 34 & 31 & 36.47 & 123 & 33.42 & & 67 & 32.21 & 87 & 35.51 & \\
\hline$\geq 65$ & 214 & 47.24 & 43 & 50.59 & 171 & 46.47 & & 110 & 52.88 & 104 & 42.45 & \\
\hline Female & 250 & 55.19 & 48 & 56.47 & 202 & 54.89 & 0.81 & 125 & 60.1 & 125 & 51.02 & 0.058 \\
\hline \multicolumn{13}{|l|}{ Race } \\
\hline Black & 399 & 88.08 & 72 & 84.71 & 327 & 88.86 & 0.334 & 185 & 88.94 & 214 & 87.35 & 1 \\
\hline White & 27 & 5.96 & 8 & 9.41 & 19 & 5.16 & & 12 & 5.77 & 15 & 6.12 & \\
\hline Others & 18 & 3.97 & 3 & 3.53 & 15 & 4.08 & & 8 & 3.85 & 10 & 4.08 & \\
\hline Unknown & 9 & 1.99 & 2 & 2.35 & 7 & 1.9 & & 3 & 1.44 & 6 & 2.45 & \\
\hline \multicolumn{13}{|l|}{ Ethnicity } \\
\hline Hispanic or Latino & 19 & 4.19 & 4 & 4.71 & 15 & 4.08 & 0.768 & 7 & 3.37 & 12 & 4.9 & 0.483 \\
\hline Not Hispanic or Latino & 424 & 93.6 & 80 & 94.12 & 344 & 93.48 & & 199 & 95.67 & 225 & 91.84 & \\
\hline Unknown & 10 & 2.21 & 1 & 1.18 & 9 & 2.45 & & 2 & 0.96 & 8 & 3.27 & \\
\hline \multicolumn{13}{|l|}{ Hypertension } \\
\hline CAD & 123 & 27.15 & 23 & 27.06 & 100 & 27.17 & 1 & 71 & 34.13 & 52 & 21.22 & 0.003 \\
\hline $\mathrm{HF}$ & 116 & 25.61 & 34 & 40 & 82 & 22.28 & 0.001 & 76 & 36.54 & 40 & 16.33 & $<0.001$ \\
\hline Diabetes & 221 & 48.79 & 54 & 63.53 & 167 & 45.38 & 0.003 & 115 & 55.29 & 106 & 43.27 & 0.011 \\
\hline Stroke & 13 & 2.87 & 6 & 7.06 & 7 & 1.9 & 0.021 & 8 & 3.85 & 5 & 2.04 & 0.273 \\
\hline AFib & 62 & 13.69 & 20 & 23.53 & 42 & 11.41 & 0.008 & 42 & 20.19 & 20 & 8.16 & $<0.001$ \\
\hline PVD & 27 & 5.96 & 5 & 5.88 & 22 & 5.98 & 1 & 20 & 9.62 & 7 & 2.86 & 0.003 \\
\hline CKD & 121 & 26.71 & 28 & 32.94 & 93 & 25.27 & 0.174 & 79 & 37.98 & 42 & 17.14 & $<0.001$ \\
\hline ESRD & 34 & 7.51 & 5 & 5.88 & 29 & 7.88 & 0.652 & 21 & 10.1 & 13 & 5.31 & 0.072 \\
\hline Cancer & 67 & 14.79 & 16 & 18.82 & 51 & 13.86 & 0.24 & 39 & 18.75 & 28 & 11.43 & 0.034 \\
\hline Obesity & 194 & 42.83 & 41 & 48.24 & 153 & 41.58 & 0.276 & 97 & 46.63 & 97 & 39.59 & 0.153 \\
\hline Morbid obesity & 80 & 17.66 & 15 & 17.65 & 65 & 17.66 & 1 & 35 & 16.83 & 45 & 18.37 & 0.712 \\
\hline \multicolumn{13}{|l|}{ Associated medication use } \\
\hline Beta blocker & 80 & 17.66 & 35 & 41.18 & 45 & 12.23 & $<0.001$ & 80 & 38.46 & 0 & 0 & $<0.001$ \\
\hline Loop diuretic & 46 & 10.15 & 16 & 18.82 & 30 & 8.15 & 0.008 & 46 & 22012 & 0 & 0 & $<0.001$ \\
\hline Spironolactone & 23 & 5.08 & 7 & 8.24 & 16 & 4.35 & 0.167 & 23 & 11.06 & 0 & 0 & $<0.001$ \\
\hline $\begin{array}{l}\text { Thiazide/thiazide-like } \\
\text { diuretic }\end{array}$ & 53 & 11.7 & 25 & 29.41 & 28 & 7.61 & $<0.001$ & 53 & 25.48 & 0 & 0 & $<0.001$ \\
\hline Other antihypertensives & 25 & 5.52 & 12 & 14.12 & 13 & 3.53 & 0.001 & 25 & 12.02 & 0 & 0 & $<0.001$ \\
\hline Statin & 88 & 19.43 & 39 & 45.88 & 49 & 13.32 & $<0.001$ & 88 & 42.31 & 0 & 0 & $<0.001$ \\
\hline
\end{tabular}

ACEI, angiotensin-converting enzyme inhibitors; ARB, angiotensin receptor blockers; CCB, calcium channel blockers.

signs, use of noninvasive positive pressure ventilation, mechanical ventilator use, and new-onset hemodialysis requirement), and clinical outcomes and data on all associated medication use (ACEI, $\mathrm{ARB}, \mathrm{CCB}$, beta blockers, loop diuretics, spironolactone, thiazide/ thiazide-like diuretics, other antihypertensive medications, and statins) were collected.

Through our institutional EPIC electronic medical record system, International Classification of Diseases (ICD)-9 and ICD-10 codes were pulled to construct comorbidity variables, and medications exposure from 14 days prior to admission date was obtained. All personal health-identifying information was deidentified, and data were extracted using an electronic coding system to maintain confidentiality. The data extraction process was subtly analyzed by experienced providers to yield precise results.

COVID-19 Outcomes in RAS Blockers

and Calcium Channel Blockers

\section{Defining Exposure to Medications of Interest and Delineating} Outcomes

We had 3 main exposures of interest: ACEI, ARB, and CCB. A complete list of different ACEI, ARB, and CCB used in the USA was pulled to avoid missing any medications exposure. Sacubitril-valsartan was categorized as an ARB. Medication exposure was defined as having refill/s of prescription of these medications within the past year and active prescriptions of these medications within the past 14 days of the date of admission. Even if the medications of interest were being held during admission for any acute issues (hypotension, sepsis, acute kidney injury, and inability to take oral medications), these patients were still included based on their medication exposure as defined above. 
The primary outcomes were to compare risk of in-hospital allcause mortality and ICU admission (as determined by mechanical ventilation need, need for noninvasive positive pressure ventilation, oxygen requirement $>6 \mathrm{~L}$, multiorgan failure syndrome, and shock) in admitted COVID-19 patients with respect to exposure to medications of interest versus in those who were not on these medications. Secondary outcomes were comparing the length of stay, 30-day readmissions, and risk of acute renal failure syndromes needing new-onset dialysis across these groups.

\section{Statistical Analysis}

For statistical analysis, we used STATA MP16 statistical software. In the first part of the study, patients were divided into subgroups based on their medication exposure regardless of indication for medication use. A total of 841 patients were included. For baseline characteristics in these patients, groups were divided into ACEI or ARB exposure versus those who were not on these medications. Similarly, the CCB exposure group was compared to those who were not on any CCB (shown in Table 1).

For the second part of the study, only hospitalized COVID-19 patients with a history of hypertension were included. A total of 453 patients out of 841 were identified. Baseline characteristics were compared in ACEI/ARB exposure in those with hypertension versus the non-ACEI/ARB group. Similarly, this was done for the CCB exposure versus the non-CCB exposure group (shown in Table 2).

For both parts of the study, baseline patient characteristics are summarized using mean and standard deviation for continuous variables and as numbers and percentages for categorical variables. Statistical differences between groups were analyzed using Fisher's exact test.

In both components of the study, analyses of outcomes were compared across ACEI users versus nonusers, ARB users versus nonusers, $\mathrm{ACEI} / \mathrm{ARB}$ users versus nonusers, $\mathrm{CCB}$ users versus nonusers, and $\mathrm{ACEI} / \mathrm{ARB}$ and $\mathrm{CCB}$ users versus nonusers. For comparison of different composite endpoints (in-hospital mortality and ICU admission), we used corresponding odds ratio for exposed group versus unexposed group with respect to particular medication/medications exposure, both unadjusted and adjusted. Multivariate logistic regression was performed to adjust for age, sex, race/ethnicity, comorbidities, and COVID-19 treatment (hydroxychloroquine, lopinavir, remdesivir, and tocilizumab) for analyzing results of outcomes.

\section{Results}

As noted, we divided our retrospective cohort study into 2 parts. For the first part, we evaluated outcomes of hospitalized COVID-19 patients based on their exposure to particular medications regardless of a personal history of hypertension. A total of 841 hospitalized patients with COVID-19 were included in this group: 111 (13.19\%) were on ACEI/ARB (ACEI users: 68, ARB users: 43) and $730(86.80 \%)$ were not on them, while 277 (32.93\%) used CCB and $564(67.06 \%)$ were not on CCB. Ninety-nine patients $(11.77 \%)$ were on both ACEI/ARB and CCB (ACE inhibitor and CCB: 59 [7.01\%]; ARB and CCB: 40
[4.75\%]). The first patient was included on March 15, 2020, and the last on June 22, 2020.

Baseline characteristics of the study groups are shown in Table 1. Users of ACEI/ARB were older than nonusers (mean age 66.1 [SD 15.4] years vs. mean age 56.6 [SD 20.3 ] years, $p<0.001)$, but the 2 groups were not significantly different in terms of sex $(p=0.685)$ and race $(p=$ 0.142 ). ACEI/ARB users were more likely to have comorbidities especially cardiovascular diseases; for example: hypertension ( 76.6 vs. $50.4 \%, p<0.001$ ), coronary artery disease (30.6 vs. $20.5 \%, p<0.02$ ), congestive heart failure ( 47.7 vs. $22.7 \%, p<0.001$ ), diabetes ( 60.4 vs. $38.5 \%$, $p<$ 0.001 ), atrial fibrillation ( 27.9 vs. $9.9 \%, p<0.001)$, and stroke ( 8.1 vs. $1.9 \%, p<0.001$ ). Users of CCB were also older than non-CCB users (mean age 64.6 [SD 15.2] years vs. mean age 54.6 [SD 21.2] years, $p<0.001)$. CCB use was somewhat higher among females ( 57.04 vs. $47.16 \%, p=$ 0.008). More than $80 \%$ patients were African American in both groups. Among CCB users, except for morbid obesity, all other comorbidities were significantly higher as shown in Table 1.

In the second part of the study, we included only those COVID-19 hospitalized patients who had a personal history of hypertension. A total of 453 patients were included. Baseline characteristics of the study group are shown in Table 2. Eighty five (18.76\%) were on ACEI/ARB (ACEI users: 68; ARB users: 43) and 368 (81.23\%) were not on them, while 208 (45.91\%) used CCB and 245 $(54.08 \%)$ were not on CCB. Seventy-three patients (16.11\%) were on both ACEI/ARB and CCB (ACE inhibitor and CCB: 42 [9.27\%]; ARB and CCB: 31 [6.62\%]).

Users of the ACEI/ARB group versus the non-ACEI/ ARB group here were not significantly different with respect to age $(p=0.33)$, sex ( $p$ value 0.81$)$, or ethnicity ( $p$ value 0.77 . More than $84 \%$ population was African American and around 55\% were female in both groups. ACEI/ARB users had higher incidence of congestive heart failure ( 40 vs. $22.3 \%, p$ 0.001), diabetes ( 63.5 vs. $45.3 \%, p$ 0.003 ), stroke ( 7.06 vs. $1.9 \%, p 0.021)$, and atrial fibrillation ( 23.5 vs. $11.4 \%, p$ 0.008).

Users of CCB were older than non-CCB users (mean age 65.0 [SD 14.8] years vs. mean age 61.7 [SD 17.3] years, $p<0.049)$. CCB use was nonsignificantly higher among females (60.1 vs. $51 \%$, p 0.06). More than $85 \%$ patients were African American in both groups. Among CCB users, incidences of CAD (34.13 vs. 21.22\%, p 0.003), CHF ( 36.54 vs. $16.33 \%, p<0.001$ ), diabetes ( 55.29 vs. $43.27 \%$, $p 0.01)$, atrial fibrillation $(20.19$ vs. $8.16 \%, p<0.001)$, PVD (9.62 vs. $2.86 \%, p 0.003$ ), and CKD stage $1-4$ (37.98 vs. $17.14 \%, p<0.001$ ) were higher. 
Table 3. Primary outcomes in all hospitalized COVID-19 patients with regard to medication exposure

\begin{tabular}{|c|c|c|c|c|}
\hline & \multicolumn{2}{|l|}{ Unadjusted } & \multicolumn{2}{|l|}{ Fully adjusted } \\
\hline & odds ratio $(95 \% \mathrm{CI})$ & $p$ value & odds ratio $(95 \% \mathrm{CI})$ & $p$ value \\
\hline \multicolumn{5}{|l|}{ All-cause in-hospital mortality } \\
\hline ACEI users versus nonusers & $1.26(0.63,2.52)$ & 0.56 & $0.58(0.24,1.36)$ & 0.21 \\
\hline ARB users versus nonusers & $1.74(0.79,3.81)$ & 0.22 & $1.11(0.44,2.78)$ & 0.82 \\
\hline ACEI/ARB users versus nonusers & $1.58(0.92,2.72)$ & 0.12 & $0.77(0.39,1.49)$ & 0.44 \\
\hline CCB users versus nonusers & $1.47(0.96,2.24)$ & 0.07 & $0.77(0.45,1.33)$ & 0.35 \\
\hline $\mathrm{ACEI} / \mathrm{ARB}$ and CCB users versus nonusers & $1.45(0.81,2.59)$ & 0.21 & $0.75(0.36,1.55)$ & 0.44 \\
\hline \multicolumn{5}{|l|}{ Risk of ICU admission } \\
\hline ACEI users versus nonusers & $1.02(0.61,1.72)$ & 0.89 & $0.67(0.37,1.20)$ & 0.18 \\
\hline ARB users versus nonusers & $1.04(0.55,1.98)$ & 0.87 & $0.61(0.29,1.27)$ & 0.19 \\
\hline ACEI/ARB users versus nonusers & $1.02(0.67,1.54)$ & 0.91 & $0.58(0.35,0.95)$ & 0.03 \\
\hline CCB users versus nonusers & $1.09(0.81,1.47)$ & 0.59 & $0.72(0.49,1.05)$ & 0.09 \\
\hline $\mathrm{ACEI} / \mathrm{ARB}$ and $\mathrm{CCB}$ users versus nonusers & $0.98(0.63,1.52)$ & 0.93 & $0.55(0.32,0.94)$ & 0.03 \\
\hline
\end{tabular}

ACEI, angiotensin-converting enzyme inhibitors; ARB, angiotensin receptor blockers; $\mathrm{CCB}$, calcium channel blockers.

In-Hospital All-Cause Mortality, ICU Admission, and Secondary Outcomes in All Hospitalized COVID-19 Patients

Table 3 shows the unadjusted and adjusted odds ratio analysis for all-cause in-hospital mortality and ICU admission in hospitalized COVID-19 patients regardless of their history of hypertension, based on exposure to medications of interest. Neither ACEI nor ARB exposure was associated with any significant effect on mortality in unadjusted or in fully adjusted analysis. Similarly, CCB use itself was not associated with any significant effect on mortality. Similarly, in those who were on both ACEI/ $\mathrm{ARB}$ and $\mathrm{CCB}$, in a fully adjusted model, there was no significant effect on in-hospital mortality (OR 0.75, 95\% CI $[0.36,1.55], p=0.44)$.

As noted in Table 3, ACEI/ARB use was not associated with reduction in incidence of ICU admission in unadjusted analysis (OR 1.02, 95\% CI $[0.67,1.54], p$ value 0.91 ); but after adjusting for age, demographics, comorbidities, and treatment for COVID-19, ACEI/ARB exposure showed $42 \%$ reduction in the risk of ICU admission (OR 0.58, 95\% CI $[0.35,0.95]$, $p$ value 0.03 ). For CCB, use was not associated with any significant reduction in ICU admission in unadjusted analysis (OR 1.09, 95\% CI [0.81, 1.47], $p$ value 0.59 ) or in the fully adjusted model (OR $0.72,95 \%$ CI $[0.49,1.05], p$ value 0.09 ).

In the group exposed to both ACEI/ARB and CCB, the unadjusted model did not show any reduction in ICU admission (OR $0.98, p$ value $0.93,95 \%$ CI $[0.63,1.52]$ ); but in the fully adjusted model, combined use of ACEI/ARB and $\mathrm{CCB}$ was associated with statistically significant (45\%) reduction in ICU admission (OR 0.55, 95\% CI $[0.32,0.94], p$ value 0.029$)$.

Neither ACEI/ARB exposure, CCB exposure, nor combined use of ACEI/ARB and CCB was associated with any significant effect on length of stay or 30-day readmission rate. But, in the fully adjusted model, ACEI/ARB exposure was associated with higher incidence of acute renal failure syndromes needing new-onset dialysis on or after the admission (OR 2.30, 95\% CI [1.09, 4.83], $p=0.028$ ).

In-Hospital All-Cause Mortality, ICU Admission, and Secondary Outcomes in Hospitalized COVID-19

Patients with a Personal History of Hypertension

Table 4 shows unadjusted and adjusted odds ratio analysis for all-cause in-hospital mortality and ICU admission in hospitalized COVID-19 patients with a personal history of hypertension, based on exposure to medications of interest. In this group of patients, as noted, $\mathrm{ARB}$ exposure or $\mathrm{CCB}$ exposure alone had no significant effect on in-hospital mortality. On the contrary, ACEI use in hospitalized COVID-19 patients with hypertension was associated with $71 \%$ reduction of in-house mortality (OR $0.29,95 \%$ CI $[0.09,0.093], p$ value 0.03 ) in the fully adjusted model. In those, who were on both ACEI/ARB and $\mathrm{CCB}$, in a fully adjusted model, there was nonsignificant reduction on in-hospital mortality (OR 0.44, 95\% CI $[0.16,1.18], p$ value 0.10$)$. 
Table 4. Primary outcomes in hospitalized COVID-19 patients with a history of hypertension with respect to medication exposure

\begin{tabular}{|c|c|c|c|c|}
\hline & \multicolumn{2}{|l|}{ Unadjusted } & \multicolumn{2}{|l|}{ Fully adjusted } \\
\hline & odds ratio $(95 \% \mathrm{CI})$ & $p$ value & odds ratio $(95 \% \mathrm{CI})$ & $p$ value \\
\hline \multicolumn{5}{|l|}{ Risk of all-cause in-hospital mortality } \\
\hline ACEI users versus nonusers & $0.66(0.26,1.67)$ & 0.51 & $0.29(0.09,0.93)$ & 0.03 \\
\hline ARB users versus nonusers & $1.78(0.75,4.22)$ & 0.19 & $1.43(0.47,4.37)$ & 0.53 \\
\hline ACEI/ARB users versus nonusers & $1.17(0.61,2.26)$ & 0.60 & $0.55(0.23,1.30)$ & 0.17 \\
\hline $\mathrm{CCB}$ users versus nonusers & $1.30(0.76,2.22)$ & 0.34 & $0.69(0.35,1.38)$ & 0.29 \\
\hline $\mathrm{ACEI} / \mathrm{ARB}$ and CCB users versus nonusers & $1.00(0.48,2.07)$ & 0.99 & $0.44(0.16,1.19)$ & 0.10 \\
\hline \multicolumn{5}{|l|}{ Risk of ICU admission } \\
\hline ACEI users versus nonusers & $0.96(0.52,1.76)$ & 0.88 & $0.67(0.34,1.35)$ & 0.26 \\
\hline ARB users versus nonusers & $0.96(0.46,2.00)$ & 0.91 & $0.68(0.29,1.59)$ & 0.38 \\
\hline ACEI/ARB users versus nonusers & $0.93(0.57,1.53)$ & 0.78 & $0.59(0.33,1.05)$ & 0.07 \\
\hline CCB users versus nonusers & $1.03(0.70,1.52)$ & 0.87 & $0.78(0.49,1.26)$ & 0.31 \\
\hline $\mathrm{ACEI} / \mathrm{ARB}$ and CCB users versus nonusers & $0.86(0.50,1.48)$ & 0.59 & $0.53(0.98,1.01)$ & 0.06 \\
\hline
\end{tabular}

ACEI, angiotensin-converting enzyme inhibitors; ARB, angiotensin receptor blockers; CCB, calcium channel blockers.

As shown in Table 4, ACEI/ARB use was associated with potentially decreased ICU admission in the fully adjusted model but did not reach statistical significance (OR $0.59,95 \%$ CI $[0.33,1.05], p$ value 0.07$)$. CCB exposure showed no significant effect on risk of ICU admissions (OR $0.78,95 \%$ CI $[0.49,1.26]$, $p$ value 0.31 ). In those who were on both $\mathrm{ACEI} / \mathrm{ARB}$ and $\mathrm{CCB}$, there was a potential trend towards a reduction in ICU admissions (OR 0.52, $95 \%$ CI $[0.27,1.02], p$ value 0.06$)$ in hospitalized COVID-19 patients with hypertension.

Neither ACEI/ARB exposure, CCB exposure, nor combined use of $A C E I / A R B$ and $C C B$ had any significant effect on 30-day readmission or length of stay even in this group. Also, ACEI/ARB exposure was not associated with significant effect on the risk for acute renal failure syndromes needing new-onset dialysis on or after admission for COVID-19 infection (OR 1.93, 95\% CI [0.77, 4.79], $p$ value 0.16 ). But when taken alone, $A R B$ exposure was associated with an increased odds of developing acute renal failure syndrome needing new-onset dialysis (OR 4.77, $95 \%$ CI $[1.44,15.85], p$ value 0.01$)$ in hospitalized COVID-19 patients with a history of hypertension.

\section{Discussion}

\section{Key Summary of Findings}

In patients hospitalized with COVID-19 infection at our tertiary care center, this retrospective study noted a trend toward less severe COVID-19 infection as noted by a reduction in the incidence of ICU admissions in those who were on ACEI/ARBs after adjusting for patient demographics, comorbidities, and COVID-19 therapeutics. In those admitted with COVID-19, who had a personal history of hypertension, ACEI exposure was associated with potentially beneficial effect on mortality after adjusting for demographics and covariates. While CCB use independently was not associated with any significant impact on outcomes in our study population, there appeared to be decreased rate of ICU admissions in those who were on both CCB and ACEI/ARB.

Overall, exposure to medications had no effect on length of stay or 30-day readmissions for the patients. Those who were on ACEI/ARB had higher chances of developing severe renal failure requiring new-onset dialysis regardless of personal history of hypertension. In those with hypertension who developed acute renal failure syndrome, ARB exposure was associated with significantly higher odds of severe renal failure syndrome needing new-onset dialysis.

\section{Comparison with Other Studies and Review of the Literature}

Evidence, till date, has failed to show any significant harmful effects of ACEI/ARB in those infected with SARS-CoV-2. Hippisley-Cox et al. [20] reported a reduced incidence of COVID-19 infection in those on ACEI/ARB overall but suggested that ARB were signifi- 
cantly less protective in the Black population than the Caucasian group; ACEI appeared less protective in the Caribbean than the Caucasian group and were associated with an increased risk of COVID-19 disease in the Black population; but neither medication was associated with an increased risk of receiving ICU care in their study. An et al. [21] suggested that treatment with ACEI/ARB was not associated with an increased risk for COVID-19 infection, while Morales et al. [22] and Christiansen et al. [23] discussed that use was neither associated with higher risk nor with any adverse outcomes in patients with COVID-19 infection. Lopes et al. [24], in their multicenter randomized trial called BRACE CORONA, aimed to evaluate whether discontinuing compared with maintaining chronic ACEI/ARB treatment among hospitalized COVID-19 patients increases days alive and out of the hospital and found no significant differences in outcomes suggesting suspending these medications was not beneficial in anyway. Similarly, Cohen et al. [25], with their randomized trial, showed that there is no effect of continuation or discontinuation of RAS blocker on hospital outcomes. In contrast, a few recent studies have pointed towards possible beneficial effects of RAS blockers in these patients. Bean et al. [26] reported a potential trend toward beneficial effect of ACEI/ARB in their study population at the multisite UK hospital; but this study only had $\sim 26 \%$ Black population. Zhang et al. [27] in their multicenter study in China showed that in-hospital use of ACEI/ARB among patients with hypertension was associated with lower risk of all-cause mortality due to COVID-19 compared with either nonuse of ACEI/ARB or use of a different class of antihypertensive agents. Yan et al. [28] found an association between ARB and reduced mortality rate in COVID-19 patients with hypertension and also suggested independent protective effects of ACEI and CCB against disease severity in elderly patients with COVID-19.

Though our study is observational and unidentified confounding factors might have contributed to our findings, the obtained results are consistent with some of the aforementioned studies showing potential beneficial effects of ACEI/ARB. Moreover, it included a predominant African American patient population who appear to suffer more severe COVID-19 infections compared to other ethnic groups [35] and in whom data with regard to medication exposure have been relatively lacking compared to Asian or Caucasian population.

Pathophysiology behind beneficial effects of renin-angiotensin-aldosterone system blockade in COVID-19 infection is still emerging. But, it has been suggested that
RAS blockers achieve this by establishing balance between ACE-2 and RAS [36]. ACE-2 is an enzyme found on the cell membrane which degrades vasoconstrictor angiotensin II (AT-II) into angiotensin 1-7 which are cardioprotective peptides [37, 38]. COVID-19 appears to either downregulate or release ACE-2 from the external membrane into the circulation system reducing its effects and causing enhancement of angiotensin II and RAS activity $[12,39]$. ACEI/ARB, with their RAS-blocking properties, can help restore balance between AT-II and Ang 1-9 and can potentially help mitigate consequences [36]. Apart from the lungs, ACE-2 is also expressed in the heart, vascular endothelium, kidneys, GI tract, etc. [40], and it has been described that ACE- 2 directly interacts with macrophages during acute inflammatory processes such as viral infections [41]. Those with CV diseases, especially hypertension, already have heightened RAS activity and thus are more prone to damage. Therefore, by keeping uncontrolled RAS activation in check, ACEI/ $\mathrm{ARB}$ are thought to play a potentially important role in reducing morbidity related to COVID-19. A retrospective review of 539 consecutive hospitalized patients with viral pneumonia indicated that continuing in-hospital use of ACEI/ARB may reduce the risk of pneumonia and death (ACEI, odds ratio 0.64 for risk of pneumonia; odds ratio 0.25 for in-hospital death; $A R B$, odds ratio 0.48 for risk of pneumonia; odds ratio 0.75 for in-hospital death) [42]. In contrast to Yan et al. [28], our study found potential beneficial effect of ACEI, rather than ARB, on mortality in hospitalized COVID-19 patients with hypertension.

$\mathrm{CCB}$ are recommended as one of the first-line agents for treatment of hypertension in African American patients. Beneficial roles of CCB in viral infections have been described over a few decades now. Studies over the years have demonstrated inhibition of influenza A virus entry as well as replication by certain CCB $[29,43,44]$. In addition, CCB also have shown to significantly decrease replication of West Nile [45], dengue [46], and Ebola virus [47]. Apart from suggesting calcium ion's role in SARS-CoV-2 virion entry, host-membrane fusion, and intracellular viral replication, Navarese et al. [30] also suggested pharmacological agents targeting ion channels may help modulate virus life cycles. In light of these findings and hypotheses, a few studies analyzing impact of CCB in COVID-19 patients have been reported.

A study from a community hospital in Brooklyn, NY, USA, reported improved mortality and a decreased risk for intubation and mechanical ventilation in hospitalized COVID-19 hypertensive patients older than 65 years of age on nifedipine/amlodipine, but this study only had 65 
patients, out of which only 21 were on these medications [32]. A study from France, using biomedical informatics in the form of natural language processing, pulled data from 39 hospitals and suggested that use of $\mathrm{CCB}$ was associated with a decreased in-hospital mortality in admitted COVID-19 patients with hypertension, but authors only adjusted for age, sex, obesity, diabetes, and cancer and not for other comorbidities or ethnicity and also did not define CCB exposure in these patients [33]. Zhang et al. [27], in their study from China, found that amlodipine was associated with a reduced case fatality rate in hospitalized COVID-19 patients, while Yan et al. [28], as noted, showed possible protective effects of CCB on COVID-19 disease severity in elderly patients. A randomized clinical trial (clinicaltrials.gov ID: NCT04351763) is also being conducted investigating the role of amiodarone and verapamil to inhibit ion channels in hospitalized patients with proven COVID-19. In our study, we did not find any significant association of independent CCB use on mortality or severity of hospitalized COVID-19 patients.

From our review of the literature, none of the studies have evaluated outcomes in hospitalized COVID-19 patients based on combined ACEI/ARB and CCB exposure. In our study, we found potentially protective effect of combined exposure to these medications on severe COVID-19 infection.

\section{Limitations}

Our study has several limitations. This was a retrospective observational study, and therefore despite controlling for various comorbidities and medications, unknown confounders might have played a role in affecting the results, and results should be interpreted with caution. Also, causal inference cannot be pointed out as it was an observational study. Though our study has yielded some results consistent with recent research data, our patient population predominantly is composed of African American patients, and thus it would be difficult to generalize the results to the population. Moreover, this study has relatively small population from a single site, and more large-scale trials which are already being conducted in this direction would give better answers. We analyzed drug classes and not individual drugs due to lack of volume otherwise. Also, we did not take into account dosage of different drugs or their duration of exposure and therefore cannot determine duration and dose-dependent effect on a particular medication if any exists. We have focused on in-hospital mortality in our analysis, and we have identified those who went to hospice care. But, 30day or 90-day effect on mortality was not calculated here due to lack of data, and this would be calculated and taken into account once more data are available to us. Data on steroids as a treatment option for severe COVID-19 infection were not available during our study period, and thus effects of steroids on mortality were excluded.

\section{Conclusion}

Our study points toward a potential trend towards a reduced risk of ICU admission in hospitalized African American patients with COVID-19 who were using ACEI or ARB. Though CCB independently were not associated with any significant effect on outcomes of hospitalized COVID-19 patients, we noted a possible beneficial effect of combined ACEI/ARB and CCB use on severity of this disease with the exception of severe renal failure. In those hospitalized COVID-19 patients (predominantly African American) with a history of hypertension, ACEI were associated with potential beneficial effects on mortality. But, our study cannot generate any causal inference, and results from ongoing clinical trials evaluating impact of the aforementioned medications on outcomes of COVID-19 patients would be of interest in the near future.

\section{Acknowledgements}

We would like to acknowledge the Center for Research Informatics (CRI) at the University of Chicago, as data from this study were provided by the Clinical Research Data Warehouse COVID-19 Data Mart maintained by the CRI at the University of Chicago. The Center for Research Informatics is funded by the Biological Sciences Division, the Institute for Translational Medicine/ CTSA (NIH ULITR000430), at the University of Chicago. We would like to specially acknowledge Dr. David Meltzer, MD, who provided general supervision of the COVID-19 hospital medicine research group at the University of Chicago.

\section{Statement of Ethics}

The research was conducted ethically in accordance with the World Medical Association Declaration of Helsinki. As noted in the manuscript, the study protocol was approved by the Institutional Review Board (IRB) Committee, and patient informed consent was waived by the committee.

\section{Conflict of Interest Statement}

The authors have no conflicts of interest to declare. 


\section{Funding Sources}

The authors received no funding for the research.

\section{Author Contributions}

Tatvam Choksi, MD, conceived, planned, and designed the research. Thomas Chen contributed to planning the research. Hui Zhang analyzed the data. Thomas Chen and Nikhil Malhotra contributed to the portions of the manuscript. Tatvam Choksi, MD, wrote the manuscript.

\section{References}

1 COVID-19 Map [Internet]. Johns Hopkins Coronavirus Resource Center. [cited 2020 Dec 17]. Available from: https://coronavirus. jhu.edu/map.html

2 Garg S, Kim L, Whitaker M, O’Halloran A, Cummings C, Holstein R, et al. Hospitalization rates and characteristics of patients hospitalized with laboratory-confirmed coronavirus disease 2019 - covid-net, 14 states, march 1-30, 2020. MMWR Morb Mortal Wkly Rep. 2020 Apr 17;69(15):458-64.

3 CDC. Covid-19 and your health [Internet]. Centers for Disease Control and Prevention. 2020 [cited 2020 Dec 17]. Available from: https: //www.cdc.gov/coronavirus/2019ncov/need-extra-precautions/people-withmedical-conditions.html

4 Pan W, Zhang J, Wang M, Ye J, Xu Y, Shen B, et al. Clinical features of covid-19 in patients with essential hypertension and the impacts of renin-angiotensin-aldosterone system inhibitors on the prognosis of covid-19 patients. Hypertension. 2020;76(3):732-41.

5 Hoffmann M, Kleine-Weber H, Schroeder S, Krüger N, Herrler T, Erichsen S, et al. Sarscov- 2 cell entry depends on ace 2 and tmprss 2 and is blocked by a clinically proven protease inhibitor. Cell. 2020;181(2):271-e8.

6 Ferrario CM, Jessup J, Chappell MC, Averill DB, Brosnihan KB, Tallant EA, et al. Effect of angiotensin-converting enzyme inhibition and angiotensin ii receptor blockers on cardiac angiotensin-converting enzyme 2 . Circulation. 2005 May 24;111(20):2605-10.

7 Soler MJ, Ye M, Wysocki J, William J, Lloveras J, Batlle D. Localization of ACE2 in the renal vasculature: amplification by angiotensin II type 1 receptor blockade using telmisartan. Am J Physiol Renal Physiol. 2009 Feb;296(2): F398-405.

8 Ishiyama Y, Gallagher PE, Averill DB, Tallant EA, Brosnihan KB, Ferrario CM. Upregulation of angiotensin-converting enzyme 2 after myocardial infarction by blockade of angiotensin II receptors. Hypertension. 2004;43(5): 970-6.

9 Fang L, Karakiulakis G, Roth M. Are patients with hypertension and diabetes mellitus at increased risk for COVID-19 infection? Lancet Respir Med. 2020 Apr;8(4):e21.

10 Wysocki J, Lores E, Ye M, Soler MJ, Batlle D. Kidney and lung ACE2 expression after an ACE inhibitor or an Ang II receptor blocker: implications for COVID-19. J Am Soc Nephrol. 2020;31(9):1941-3.
11 Zheng YY, Ma YT, Zhang JY, Xie X. COVID-19 and the cardiovascular system. Nat Rev Cardiol. 2020;17(5):259-60.

12 Vaduganathan M, Vardeny O, Michel T, McMurray JJV, Pfeffer MA, Solomon SD. Reninangiotensin-aldosterone system inhibitors in patients with covid-19. N Engl J Med. 2020; 382(17):1653-9.

13 Kuster GM, Pfister O, Burkard T, Zhou Q Twerenbold R, Haaf P, et al. SARS-CoV2: should inhibitors of the renin-angiotensin system be withdrawn in patients with $\mathrm{CO}$ VID-19? Eur Heart J. 2020;41(19):1801-3.

14 Tomasoni D, Italia L, Adamo M, Inciardi RM, Lombardi CM, Solomon SD, et al. COVID-19 and heart failure: from infection to inflammation and angiotensin II stimulation. Searching for evidence from a new disease. Eur J Heart Fail. 2020 Jun;22(6):957-66.

15 Danser AHJ, Epstein M, Batlle D. Renin-angiotensin system blockers and the covid-19 pandemic: at present there is no evidence to abandon renin-angiotensin system blockers. Hypertension. 2020;75(6):1382-5.

16 Fosbøl EL, Butt JH, Østergaard L, Andersson C, Selmer C, Kragholm K, et al. Association of angiotensin-converting enzyme inhibitor or angiotensin receptor blocker use with covid-19 diagnosis and mortality. JAMA. 2020 Jul 14;324(2):168-77.

17 Mancia G, Rea F, Ludergnani M, Apolone G, Corrao G. Renin-angiotensin-aldosterone system blockers and the risk of covid-19. N Engl J Med. 2020;382(25):2431-40.

18 Liu X, Long C, Xiong Q, Chen C, Ma J, Su Y, et al. Association of angiotensin converting enzyme inhibitors and angiotensin II receptor blockers with risk of COVID-19, inflammation level, severity, and death in patients with COVID-19: a rapid systematic review and meta-analysis. Clin Cardiol. 2020 Aug 5; $10.1002 /$ clc. 23421

19 Shah P, Owens J, Franklin J, Jani Y, Kumar A, Doshi R. Baseline use of angiotensin-converting enzyme inhibitor/AT1 blocker and outcomes in hospitalized coronavirus disease 2019 African-American patients. J Hypertens. 2020;38(12):2537-41.

20 Hippisley-Cox J, Young D, Coupland C, Channon KM, Tan PS, Harrison DA, et al. Risk of severe COVID-19 disease with ACE inhibitors and angiotensin receptor blockers: cohort study including 8.3 million people. Heart. 2020;106(19):1503-11.
21 An J, Wei R, Zhou H, Luong TQ, Gould MK, Mefford MT, et al. Angiotensin-converting enzyme inhibitors or angiotensin receptor blockers use and covid-19 infection among 824,650 patients with hypertension from a us integrated healthcare system. J Am Heart Assoc. 2021 Dec 14;10(3):e019669.

22 Morales DR, Conover MM, You SC, Pratt N, Kostka K, Duarte-Salles T, et al. Renin-angiotensin system blockers and susceptibility to COVID-19: an international, open science, cohort analysis. Lancet Digit Health. 2021 Feb;3(2):e98-114.

23 Christiansen CF, Pottegård A, Heide-Jørgensen U, Bodilsen J, Søgaard OS, Maeng M, et al. SARS-CoV-2 infection and adverse outcomes in users of ACE inhibitors and angiotensin-receptor blockers: a nationwide casecontrol and cohort analysis. Thorax. $2020 \mathrm{Dec}$ 8. E-pub ahead of print.

24 Lopes RD, Macedo AVS, de Barros E Silva PGM, Moll-Bernardes RJ, Dos Santos TM, Mazza L, et al. Effect of discontinuing versus continuing angiotensin-converting enzyme inhibitors and angiotensin ii receptor blockers on days alive and out of the hospital in patients admitted with covid-19: a randomized clinical trial. JAMA. 2021 Jan 19;325(3): 254-64.

25 Cohen JB, Hanff TC, William P, Sweitzer N, Rosado-Santander NR, Medina C, et al. Continuation versus discontinuation of renin-angiotensin system inhibitors in patients admitted to hospital with COVID-19: a prospective, randomised, open-label trial. Lancet Respir Med. 2021 Jan 7:221.

26 Bean DM, Kraljevic Z, Searle T, Bendayan R, Kevin O, Pickles A, et al. Angiotensin-converting enzyme inhibitors and angiotensin II receptor blockers are not associated with severe COVID-19 infection in a multi-site UK acute hospital trust. Eur J Heart Fail. 2020 Jun; 22(6):967-74.

27 Zhang P, Zhu L, Cai J, Lei F, Qin JJ, Xie J, et al. Association of inpatient use of angiotensin-converting enzyme inhibitors and angiotensin ii receptor blockers with mortality among patients with hypertension hospitalized with covid-19. Circ Res. 2020 05;126(12): 1671-81.

28 Yan F, Huang F, Xu J, Yang P, Qin Y, Lv J, et al. Antihypertensive drugs are associated with reduced fatal outcomes and improved clinical characteristics in elderly COVID-19 patients. Cell Discov. 2020 Oct 29;6(1):77. 
29 COVID-19: Is it time to revisit the research on calcium channel drug targets? [Internet]. European Medical Journal. 2020 [cited 2020 Dec 17]. Available from: https://www.emireviews. com/diabetes/article/covid-19-is-it-time-torevisit-the-research-on-calcium-channeldrug-targets/

30 Navarese EP, Musci RL, Frediani L, Gurbel PA, Kubica J. Ion channel inhibition against COVID-19: a novel target for clinical investigation. Cardiol J. 2020;27(4):421-4.

31 Zhang LK, Sun Y, Zeng H, Wang Q, Jiang X, Shang WJ, et al. Calcium channel blocker amlodipine besylate therapy is associated with reduced case fatality rate of COVID-19 patients with hypertension. Cell Discov. 2020 Dec 22;6(1):96.

32 Solaimanzadeh I. Nifedipine and amlodipine are associated with improved mortality and decreased risk for intubation and mechanical ventilation in elderly patients hospitalized for covid-19. Cureus. 2020 May 12;12(5):e8069.

33 Neuraz A, Lerner I, Digan W, Paris N, Tsopra $\mathrm{R}$, Rogier A, et al. Natural language processing for rapid response to emergent diseases: case study of calcium channel blockers and hypertension in the covid-19 pandemic. J Med Internet Res. 2020 Aug 14;22(8):e20773.

34 CDC. Coronavirus Disease 2019 (COVID-19) [Internet]. Centers for Disease Control and Prevention. 2020 [cited 2020 Dec 17]. Available from: https://www.cdc.gov/ coronavirus/2019-ncov/covid-data/investigations-discovery/hospitalization-death-byrace-ethnicity.html.
35 Golestaneh L, Neugarten J, Fisher M, Billett $\mathrm{HH}$, Gil MR, Johns T, et al. The association of race and COVID-19 mortality. EClinicalMedicine. 2020 Aug;25:100455.

36 Bloch MJ. Renin-angiotensin system blockade in covid-19: good, bad, or indifferent? J Am Coll Cardiol. 2020 21;76(3):277-9.

37 Santos RAS, Sampaio WO, Alzamora AC, Motta-Santos D, Alenina N, Bader M, et al. The ace2/angiotensin-(1-7)/MAS axis of the renin-angiotensin system: focus on angiotensin-(1-7). Physiol Rev. 2018 01;98(1): 505-53.

38 Esc guidance for the diagnosis and management of $\mathrm{cv}$ disease during the covid-19 pandemic [Internet]. [cited 2020 Dec 17]. Available from: https://www.escardio.org/Education/COVID-19-and-Cardiology/ ESC-COVID-19-Guidance.

39 Verdecchia P, Cavallini C, Spanevello A, Angeli $\mathrm{F}$. The pivotal link between ACE2 deficiency and SARS-CoV-2 infection. Eur J Intern Med. 2020;76:14-20.

40 Hamming I, Timens W, Bulthuis ML, Lely AT, Navis G, van Goor H. Tissue distribution of ACE2 protein, the functional receptor for SARS coronavirus. A first step in understanding SARS pathogenesis. J Pathol. 2004 Jun; 203(2):631-7.
41 Abassi Z, Knaney Y, Karram T, Heyman SN. The lung macrophage in sars-cov-2 infection: a friend or a foe? Front Immunol. 2020;11: 1312.

42 Henry C, Zaizafoun M, Stock E, Ghamande S, Arroliga AC, White HD. Impact of angiotensin-converting enzyme inhibitors and statins on viral pneumonia. Proc. 2018 Oct;31(4): $419-23$.

43 Nugent KM, Shanley JD. Verapamil inhibits influenza A virus replication. Arch Virol. 1984;81(1-2):163-70.

44 Fujioka Y, Nishide S, Ose T, Suzuki T, Kato I, Fukuhara $\mathrm{H}$, et al. A sialylated voltage-dependent ca2+ channel binds hemagglutinin and mediates influenza a virus entry into mammalian cells. Cell Host Microbe. 2018 13; 23(6):809.

45 Scherbik SV, Brinton MA. Virus-induced $\mathrm{Ca} 2+$ influx extends survival of west nile virus-infected cells. J Virol. 2010 Sep;84(17): 8721-31.

46 Dionicio CL, Peña F, Constantino-Jonapa LA, Vazquez C, Yocupicio-Monroy M, Rosales R, et al. Dengue virus induced changes in $\mathrm{Ca} 2+$ homeostasis in human hepatic cells that favor the viral replicative cycle. Virus Res. 2018 02;245:17-28.

47 Johansen LM, DeWald LE, Shoemaker CJ, Hoffstrom BG, Lear-Rooney CM, Stossel A, et al. A screen of approved drugs and molecular probes identifies therapeutics with anti-Ebola virus activity. Sci Transl Med. 2015;7(290): 290 ra89. 